The University of Southern Mississippi

The Aquila Digital Community

Faculty Publications

$9-1-2007$

\title{
Experimentally Constrained Molecular Relaxation: The Case of Hydrogenated Amorphous Silicon
}

\author{
Parthapratim Biswas \\ University of Southern Mississippi, partha.biswas@usm.edu \\ Raymond Atta-Fynn \\ University of Texas at Arlington \\ D. A. Drabold \\ Ohio University
}

Follow this and additional works at: https://aquila.usm.edu/fac_pubs

Part of the Physics Commons

\section{Recommended Citation}

Biswas, P., Atta-Fynn, R., Drabold, D. (2007). Experimentally Constrained Molecular Relaxation: The Case of Hydrogenated Amorphous Silicon. Physical Review B, 76(12).

Available at: https://aquila.usm.edu/fac_pubs/1930

This Article is brought to you for free and open access by The Aquila Digital Community. It has been accepted for inclusion in Faculty Publications by an authorized administrator of The Aquila Digital Community. For more information, please contact Joshua.Cromwell@usm.edu. 


\title{
Experimentally constrained molecular relaxation: The case of hydrogenated amorphous silicon
}

\author{
Parthapratim Biswas* \\ Department of Physics and Astronomy, The University of Southern Mississippi, Hattiesburg, Mississippi 39401, USA
}

Raymond Atta-Fynn ${ }^{\dagger}$

Department of Physics and Astronomy, The University of Texas at Arlington, Arlington, Texas 76019, USA

D. A. Drabold

Department of Physics and Astronomy, Ohio University, Athens, Ohio 45701, USA

(Received 13 February 2007; revised manuscript received 10 August 2007; published 25 September 2007)

\begin{abstract}
We have extended our experimentally constrained molecular relaxation technique [P. Biswas et al., Phys. Rev. B 71, 54204 (2005)] to hydrogenated amorphous silicon: a 540-atom model with 7.4\% hydrogen and a 611-atom model with $22 \%$ hydrogen were constructed. Starting from a random configuration, using physically relevant constraints, $a b$ initio interactions, and the experimental static structure factor, we construct realistic models of hydrogenated amorphous silicon. Our models confirm the presence of a high-frequency localized band in the vibrational density of states due to Si-H vibration that has been observed in recent vibrational transient grating measurements on plasma enhanced chemical vapor deposited films of hydrogenated amorphous silicon.
\end{abstract}

DOI: 10.1103/PhysRevB.76.125210

PACS number(s): 71.23.Cq, 71.15.Mb, 71.23.An

Ideal models of materials must be consistent with known experimentally determined attributes and must also be near a minimum of an accurate energy functional. "Informationbased" methods such as the reverse Monte Carlo (RMC) method $^{1-3}$ produce models in agreement with experiment (typically a structural measurement), though often discrepant with chemical and bonding considerations, while molecular dynamics (MD) simulations suffer from artifacts of overrapid quenches and small system sizes. To jointly satisfy these approaches, we introduced the experimentally constrained molecular relaxation (ECMR) method for amorphous materials and applied it with success to the complex binary glass $\mathrm{GeSe}_{2}{ }^{4}$ ' Intriguing related ideas based upon Bayesian methods are emerging in studies of biomolecules, ${ }^{5}$ and our scheme is also reminiscent of the empirical potential structure refinement (EPSR) approach. ${ }^{6}$

In this paper, we extend this approach to hydrogenated amorphous silicon $(a-\mathrm{Si}: \mathrm{H})$, a key electronic material with myriad applications and a great many fundamental puzzles associated with structure, hydrogen dynamics, and properties under illumination. ${ }^{7}$ Owing to the complex nature of bonding between $\mathrm{Si}$ and $\mathrm{H}$, a straightforward application of Monte Carlo or MD simulation encounters difficulty in producing experimentally realistic models of hydrogenated amorphous silicon.

Conventional approaches to modeling $a$-Si:H can be divided into two categories: static and dynamic. In the static approach $^{8-11}$ a set of predetermined geometric and chemical rules are employed to minimize the total energy either by Monte Carlo or some other minimization scheme, such as the conjugate gradient method. The so-called Wooten, Winer, and Weaire (WWW) ${ }^{12}$ algorithm is the classic example of such a technique, and uses specific Monte Carlo moves (the WWW bond switch) to produce excellent continuous random network (CRN) models of amorphous silicon. The network is then hydrogenated after creating dangling-bond defects (threefold sites) and passivating the dangling bond with $\mathrm{H}$, or by breaking a bond between a fivefold and fourfold coordinated atom and again repairing the threefold site with $\mathrm{H}$ with a suitable structural relaxation. While this approach produces models that exhibit good agreement with experiments, some of the troubling issues that warrant further investigation include distribution of hydrogen atoms and the amount of strain present in the network. In the dynamic approach ${ }^{13-15}$ a mixture of silicon and hydrogen is rapidly cooled from the melt via first-principles molecular dynamics simulation. The $\mathrm{Si}-\mathrm{H}$ interaction can be taken into account conveniently within the quantum mechanical framework, but problems arise from the short time scale and prohibitively expensive scaling of computer time with the system size. The presence of $\mathrm{H}$ atoms requires a relatively small time step during $\mathrm{MD}$ runs limiting total simulation time to few picoseconds that is inadequate to producing high-quality (small defect concentration), strain-free networks. Large scale realistic modeling of $a-\mathrm{Si}: \mathrm{H}$ is therefore impractical with MD alone. ${ }^{16}$

Reverse Monte Carlo is a popular method used to efficiently generate structural models in agreement with experiments, as discussed elsewhere. ${ }^{1-3}$ RMC readily generates an ensemble of structures but only a subset, possibly a miniscule subset, are physically meaningful. In RMC, one usually remedies this problem by adding topological and/or chemical constraints $^{3}$ but the constrained minimization is difficult within a simple Monte Carlo scheme. The way out of this dilemma is to identify constraints that are hierarchically most important along with experimental information and to merge this with either first-principles or a suitable classical force field. In a recent paper we have demonstrated that a judicious combination of these two can constrain the structure to evolve on the multidimensional energy surface correctly, which also is consistent with experimental data space. ${ }^{4,17}$

In this paper, we extend the idea of experimentally constrained molecular relaxation (ECMR) to model $a$-Si: $\mathrm{H}$. The approach is to build models by incorporating experimental information explicitly in the beginning, and to then relax 
such a starting structure to an energy minimum. In our first implementation of the method ${ }^{4}$ we self-consistently iterated the RMC and relaxation steps until convergence was obtained. In the present work, we implement this as a two-step process employing reverse Monte Carlo (RMC) followed by first-principles molecular dynamics relaxation. Only one iteration of the ECMR loop is required as we discuss below. By construction, the models we obtain satisfy the common sense criterion that a "good" model must jointly match experiments and be near to a minimum of a trusted energy functional. Their subsequent value (and validation) depends upon their predictive power for observables not employed in the construction of the models. The RMC scheme implemented in this work can be found in Ref. 3. Atoms are randomly packed into a cubic supercell using periodic boundary conditions, subject to the constraint that no Si pair can be closer than $2 \AA$. A quadratic cost or penalty function $\Pi$ is introduced in such a way that if minimized to zero, the experimental radial distribution function of amorphous silicon is exactly reproduced and a set of suitable constraints that describes the chemical and geometric properties (experimental information on the mean and RMS variation of bond angles, four coordination) of the system is also enforced. ${ }^{18,28,29}$ Naturally, it is impossible to find a set of coordinates that make $\Pi=0$, but it is possible to obtain models consistent within noise and systematic limitations (such as finite-size effects) with the experimental information and constraints. This implies a small but finite $\Pi$.

The resulting configuration obtained from RMC relaxation is a 500-atom CRN having a nonuniform distribution of coordination defects, with dangling bonds being the dominant defect. The average bond angle of the CRN is found to be $109.3^{\circ}$ with a root-mean-square (RMS) deviation of $12.6^{\circ}$. The number of fourfold coordinated atoms is found to be $88 \%$ while the remaining $12 \%$ consists of threefold and fivefold coordinated atoms.

The next step toward building a configuration of $a-\mathrm{Si}: \mathrm{H}$ is to hydrogenate the RMC-generated CRN. The hydrogenation proceeds following a scheme which is similar but not identical to that proposed by Holender and Morgan. ${ }^{10} \mathrm{We}$ generate two configurations of $a-\mathrm{Si}: \mathrm{H}$ starting from the 500atom CRN following two different hydrogenation schemes.

The first scheme involves passivating only the dangling bonds while in the second both the dangling and floating bonds (fivefold sites) are removed. For the 540-atom model, the dangling bonds are identified and passivated by placing a $\mathrm{H}$ atom at a distance of 1.45 to $1.65 \AA$, along the direction vector opposite to the sum of the three vectors connecting the central atom and its three neighbors. The floating bonds are kept undisturbed to minimize hydrogen content. The density of the $a-\mathrm{Si}: \mathrm{H}$ supercell is adjusted to experimental value once all the dangling bonds are passivated. The configuration obtained after hydrogenation has defect concentration of approximately $2 \%$, which is due to floating bonds left undisturbed during hydrogenation.

For the 611-atom model, we remove all the threefold and fivefold coordinated atoms. To remove a floating bond, we look for the nearest neighbor of the central atom that has a lowest coordination number. If a floating bond has neighbors which are all fourfold, we choose the neighbor which is far- thest from the central atom. We also check for pairs of atoms which are both fivefold coordinated and nearest neighbors of each other. In this case, we break the bond between two fivefold atoms by increasing the distance beyond the cutoff $2.8 \AA$. The second scheme evidently adds more hydrogen to the network but produces completely defect-free configurations.

Once the hydrogenation is completed, we relax the configuration until the largest atomic force acting on atoms is less than or equal to $0.007 \mathrm{eV} / \AA$. The relaxation is performed using SIESTA ${ }^{19}$ which is a local basis, first-principles density functional code. Because of large system size, we employ the Harris functional approach, ${ }^{20}$ which employs a linearized form of the Kohn-Sham equations. For silicon atoms we use minimal single- $\zeta$ (SZ) basis orbitals while for hydrogen atoms, a double- $\zeta$ basis with polarization orbitals (DZP) is needed to properly represent the $\mathrm{H}$ interactions. ${ }^{21}$ The effect of basis dependence on electronic structure of amoprhous silicon has been discussed at length in Ref. 22 .

The process of "relax and clean" goes on until the defect concentration is found to be reasonably low $(\leqslant 3 \%)$. For the 611 atom, the iteration converges after a few steps while for the 540-atom model we keep going until we obtain a configuration which is not only relaxed but also has a small defect concentration. The procedure finally yields a 540atom model with $2.9 \%$ defect concentration-1.9\% fivefold, $0.3 \%$ threefold, and $0.7 \%$ twofold. The average coordination number of the network is found to be 3.98. To the best of our knowledge, this 540-atom model is possibly the largest device quality model (obtained via first-principles) of $a-\mathrm{Si}: \mathrm{H}$ (7.4\% hydrogen) with defect concentration as low as $2.9 \%$. The 611-atom model is found to be free from any defect with the exception of a single twofold $\mathrm{Si}$ atom, and has $22 \%$ of the hydrogen.

In Fig. 1 we present the partial pair correlation functions along with experimental data reported by Laaziri et al. ${ }^{23} \mathrm{Al}-$ though the information on the Si-Si structure factor has been "built into" our starting RMC-generated CRN, it is important to ensure that the hydrogenation and relaxation does not introduce any changes in $\mathrm{Si}-\mathrm{Si}$ pair correlation. It is clear from Fig. 1 that the Si-Si pair correlation from our model agrees closely with that obtained from experiment. ${ }^{23}$ It is to be noted that the experimental data are for pure amorphous silicon, whereas the Si-Si partial pair correlation function in Fig. 1 is computed in hydrogenated environment. This possibly explains the difference in peak height of our result with the experimental data and the results obtained in Ref. 24. Apart from this slight deviation, our result matches with the experimental data reasonably well. This indicates that as the system evolves via ECMR, it moves progressively toward a more favorable configuration consistent with both total energy and experimental data.

In Fig. 2, the vibrational density of states (VDOS) is plotted for both models. The VDOS is computed by diagonalizing the dynamical matrix, which is obtained from the force constant matrix. The force constant matrix is approximated with finite differences obtained from $6 \mathrm{~N}$ calculations of forces on all atoms for suitably chosen small displacements from the equilibrium conformation. 


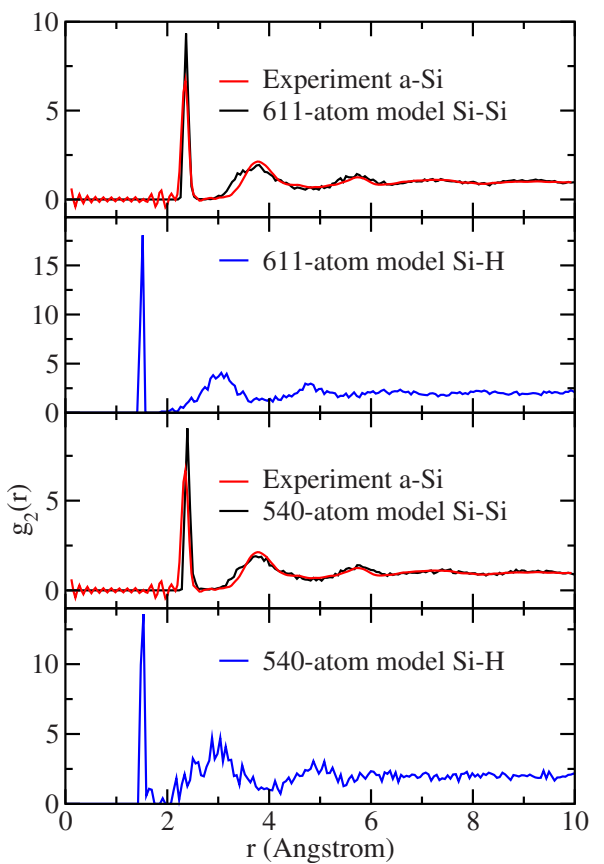

FIG. 1. (Color online) The partial pair correlation functions between $\mathrm{Si}-\mathrm{Si}$ and $\mathrm{Si}-\mathrm{H}$ for the two models of $a-\mathrm{Si}: \mathrm{H}$ simulated in this work. The experimental data for $\mathrm{Si}-\mathrm{Si}$ partial are also shown in the figure (red/gray) for comparison with our results.

Since the eigenvalues of the dynamical matrix are given by the square of the angular frequency, one can construct the density distribution of the frequencies from a knowledge of the eigenvalues. The acoustic and the optical peaks appear at correct position (22.0 and $61.0 \mathrm{meV}$, respectively) which is in good agreement with the experiment data reported by Kamitakahara et al..$^{25}$ and is also shown in the figure. Both the models show an excess of high-frequency modes which are associated with hydrogen atoms. Such high-frequency modes have been observed in the experimental vibrational spectrum of hydrogenated $a$-Si. ${ }^{26}$ Of particular importance are the vibrational modes around $250 \mathrm{meV}\left(\approx 2000 \mathrm{~cm}^{-1}\right)$. Rella et al. have studied the localization of $\mathrm{Si}-\mathrm{H}$ stretch vibration in amorphous silicon using vibrational transient grating measurement, and observed highly localized modes around $2000 \mathrm{~cm}^{-1} .{ }^{26,27}$ The presence of this high-frequency vibrational band adds further credibility to our model. To characterize the spatial extent of the vibrational states we have calculated the inverse participation ratio (IPR) of the states in this band obtained from the dynamical matrix. The IPR for a normalized state $j$ is defined as

$$
I=\sum_{i=1}^{N}\left(\Phi_{i}^{j} \Phi_{i}^{j}\right)^{2}
$$

and provides a measure of the inverse of the number of sites associated with a state. Here $\Phi^{j}$ is a normal mode eigenvector and $N$ is the number of atoms (molecules in a nonmonoatomic system) in the system. For an ideally localized state, only one atom (molecule) contributes to the vibration giving $I=1$, whereas for a uniformly extended state we have $I$

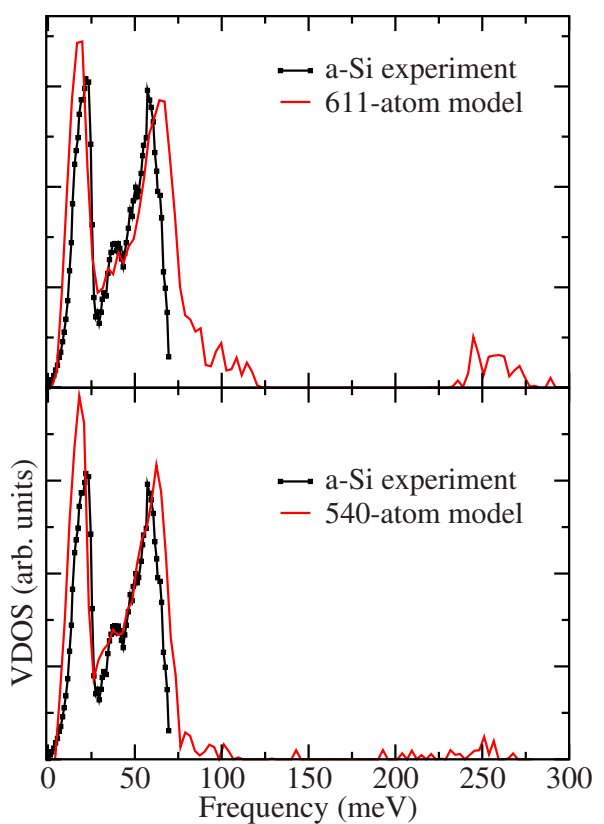

FIG. 2. (Color online) Vibrational density of states for the two models of $a-\mathrm{Si}: \mathrm{H}$. The upper and the lower panels stand for 611and 540-atom (red/gray) models, respectively. The experimental data for (unhydrogenated) amorphous Si from neutron diffraction are also plotted for comparison (black).

$=1 / N$. In Fig. 3 we have plotted the inverse participation ratio along with the vibrational density of states for the 540atom model. The localized nature of the high-frequency vibrational band is clearly visible from the plot. Our preliminary study indicates that this vibrational band is due to isolated localized states of $\mathrm{Si}-\mathrm{H}$ vibrations, and the vibrational modes correspond to frequency around $2000 \mathrm{~cm}^{-1}$ (250 meV in Fig. 3) which appear to be very similar to the character of a Si-H stretching mode. However, a complete analysis of the nature of the vibration requires a selfconsistent relaxation of the structure and an accurate determination of vibrational frequencies and eigenvectors. Investigations are presently being carried out to study the nature of vibration modes in this band.

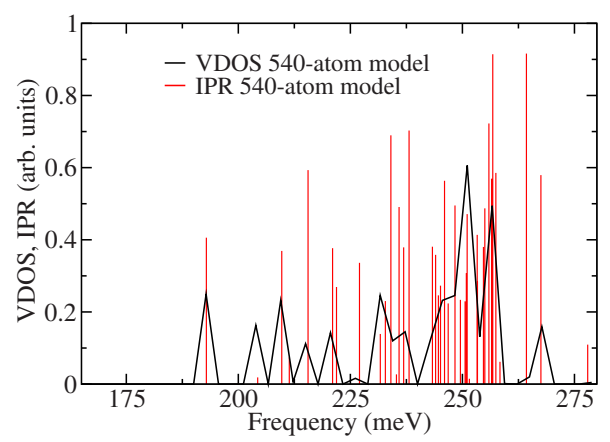

FIG. 3. (Color online) The inverse participation ratio (red/gray) of vibrational modes along with their density of states (black) for 540 -atom model. The localized nature of the high-frequency band is clear from the large inverse participation ratio. The VDOS is shown as a guide to the eyes and is scaled for plotting. 


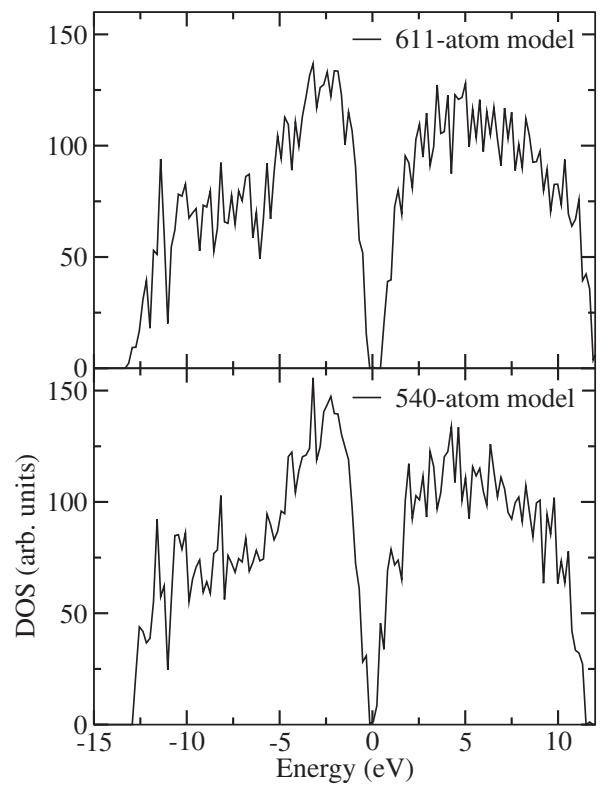

FIG. 4. The electronic density of states for the two models using Harris functional and local density approximation (LDA). The Hamiltonians for the models are constructed using single- $\zeta$ and double- $\zeta$-polarized bases for $\mathrm{Si}$ and $\mathrm{H}$ atoms, respectively.

Finally, in Fig. 4 we have plotted the electronic density of states of the models obtained from SIESTA using the local density approximation. Both the models show the presence of a clean gap in the spectrum. This electronic gap is very sensitive to the presence of defect states and other imperfections present in the model. For the 540-atom model, the electronic gap is slightly narrower than its 611-atom counterpart which is quite expected because of $2.9 \%$ concentration of defects compared to a single defect in the 611-atom model. There are no localized midgap states and a few band-tail states in the 540-atom model. These tail states are generally considered to be associated with the presence of disorder in bond length and bond angle distribution. Recent work by Bernstein et al. have indicated that the width of the bond length distribution can affect the density of states in or near the band edge region. ${ }^{24}$ We believe that further tuning of electronic density of states is possible via prolonged relaxation using our experimental constrained molecular relax-
TABLE I. Summary of existing bulk models ( $>100$ atoms) of $a-\mathrm{Si}: \mathrm{H}$.

\begin{tabular}{lccc}
\hline \hline Authors (yr) & Model size & $\%$ of $\mathrm{H}$ & Method \\
\hline ML (1991) (Ref. 8) & 270 & 16.0 & Static \\
HMJ (1993) (Ref. 10) & 2025 & 23.0 & Static+dynamic \\
TA (1996) (Ref. 11) & 242 & 11.0 & Dynamic \\
KUF (1999) (Ref. 15) & 128 & 6.25 & Dynamic \\
Present work (2007) & $540(611)$ & $7.4(22)$ & ECMR \\
\hline \hline
\end{tabular}

ation (ECMR) method ${ }^{4}$ which we have recently developed and applied to glassy $\mathrm{GeSe}_{2}$.

In conclusion, we apply ECMR to hydrogenated amorphous silicon. The importance of this approach is its simultaneous use of both experimental information and the information implicit in an accurate energy functional. We apply this method to produce two large models of $a-\mathrm{Si}: \mathrm{H}$. The structural, vibrational, and electronic properties of the models agree very well with experiments. We show that the method is fast and is capable of generating device quality $a$-Si: $\mathrm{H}$ models with low defect concentration. A comparison with existing models (see Table I) obtained by other researchers clearly reveals the fact that the method benefits from both static and dynamic approaches-it can handle a large number of atoms (a characteristic feature of the static approach) and at the same time producing highly relaxed configuration with average force on atoms as small as few meV/A. A remarkable feature of the models is that they produce a high-frequency localized vibrational band around $250 \mathrm{meV}$ which has been observed experimentally. Analysis of eigenvectors corresponding to this and other nearby modes suggests the models are localized. The models generated in this work can be used as a starting point for an indepth study of properties of $\mathrm{H}$ in amorphous silicon using a fully self-consistent calculations. The approach is very general and has the potential to impact other complex materials.

D.A.D. thanks the NSF for support under Grants Nos. DMR 0605890 and 0600073, and the ARO under Grant No. MURI W911-NF-06-2-0026. P.B. acknowledges the support of the University of Southern Mississippi under Grant No. DE00945. We thank one of the referees for pointing out Refs. 24, 26, and 27

\footnotetext{
*Partha.Biswas@usm.edu

†attafynn@uta.edu

¥drabold@ohio.edu

${ }^{1}$ O. Gereben and L. Pusztai, Phys. Rev. B 50, 14136 (1994).

${ }^{2}$ R. L. McGreevy, J. Phys.: Condens. Matter 13, R877 (2001).

${ }^{3}$ P. Biswas, R. Atta-Fynn, and D. A. Drabold, Phys. Rev. B 69, 195207 (2004).

${ }^{4}$ P. Biswas, D. N. Tafen, and D. A. Drabold, Phys. Rev. B 71, 054204 (2005).

${ }^{5}$ M. Haybeck, W. Rieping, and M. Nilges, Proc. Natl. Acad. Sci.
}

U.S.A. 103, 1756 (2006).

${ }^{6}$ A. K. Soper, Phys. Rev. B 72, 104204 (2005).

${ }^{7}$ R. A. Street, Hydrogenated Amorphous Silicon (Cambridge University Press, Cambridge, 1991), pp. 211-363.

${ }^{8}$ N. Mousseau and L. J. Lewis, Phys. Rev. B 43, 9810 (1991); 41, 3702 (1990).

${ }^{9}$ B. J. Min, Y. H. Lee, C. Z. Wang, C. T. Chan, and K. M. Ho, Phys. Rev. B 45, 6839 (1992).

${ }^{10}$ J. M. Holender, G. J. Morgan, and R. Jones, Phys. Rev. B 47, 3991 (1993). 
${ }^{11}$ B. Tuttle and J. B. Adams, Phys. Rev. B 53, 16265 (1996).

${ }^{12}$ F. Wooten, K. Winer, and D. Weaire, Phys. Rev. Lett. 54, 1392 (1985).

${ }^{13}$ D. A. Drabold, P. A. Fedders, S. Klemm, and O. F. Sankey, Phys. Rev. Lett. 67, 2179 (1991).

${ }^{14}$ F. Buda, G. L. Chiarotti, R. Car, and M. Parrinello, Phys. Rev. B 44, 5908 (1991).

${ }^{15}$ P. Klein, H. M. Urbassek, and T. Frauenheim, Phys. Rev. B 60, 5478 (1999).

${ }^{16}$ J. Robertson, J. Non-Cryst. Solids 79, 266 (2000).

${ }^{17}$ P. Biswas, D. N. Tafen, R. Atta-Fynn, and D. A. Drabold, J. Phys.: Condens. Matter 16, S5173 (2004).

${ }^{18}$ The zero-point motion of the nuclei is not taken into account in our approach. At room temperature, the effect on $\mathrm{Si}$ atoms can be neglected other than an additional broadening of the first peak of the radial distribution function. The effect on $\mathrm{H}$ atoms may be nontrivial, which can be addressed via path integral quantum Monte Carlo at high temperature or via harmonic approximation at low temperature as discussed in Refs. 28 and 29, respectively.

${ }^{19}$ P. Ordejón, E. Artacho, and J. M. Soler, Phys. Rev. B 53, R10441 (1996).

${ }^{20}$ J. Harris, Phys. Rev. B 31, 1770 (1985).
${ }^{21}$ Because of the heavy computational nature of the problem, we have not studied the role of full self-consistency and the basis dependence of Si-H bonding in our present work. Such issues might be important in understanding the electronic structure and dynamics of $\mathrm{H}$ atoms and will be addressed in a separate communication. For a discussion on $a$-Si, see Ref. 22.

${ }^{22}$ R. Atta-Fynn, P. Biswas, P. Ordejon, and D. A. Drabold, Phys. Rev. B 69, 085207 (2004).

${ }^{23}$ K. Laaziri, S. Kycia, S. Roorda, M. Chicoine, J. L. Robertson, J. Wang, and S. C. Moss, Phys. Rev. Lett. 82, 3460 (1999).

${ }^{24}$ N. Bernstein, J. L. Feldman, and M. Fornari, Phys. Rev. B 74, 205202 (2006).

${ }^{25}$ W. A. Kamitakahara, H. R. Shanks, J. F. McClelland, U. Buchenau, F. Gompf, and L. Pintschovius, Phys. Rev. Lett. 52, 644 (1984).

${ }^{26}$ M. van der Voort, C. W. Rella, L. F. G. van der Meer, A. V. Akimov, and J. I. Dijkhuis, Phys. Rev. Lett. 84, 1236 (2000).

${ }^{27}$ C. W. Rella, M. van der Voort, A. V. Akimov, A. F. G. van der Meer, and J. I. Dijkhuis, Appl. Phys. Lett. 75, 2945 (1999).

${ }^{28}$ C. P. Herrero, Europhys. Lett. 44, 734 (1998).

${ }^{29}$ J. L. Feldman, N. Bernstein, and D. A. Papaconstantopoulos, J. Phys.: Condens. Matter 16, S5165 (2004). 